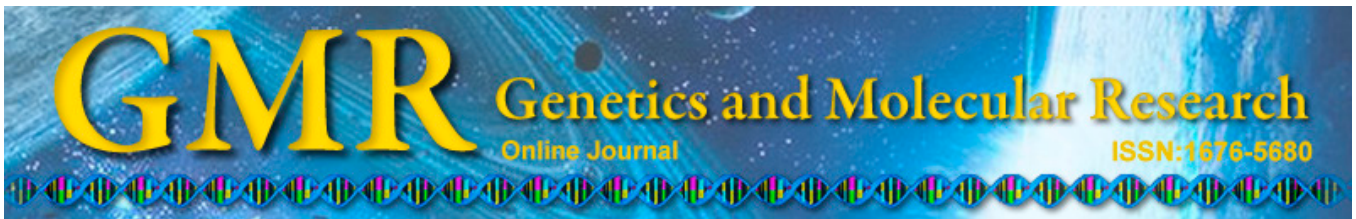

\title{
Differentiation-inducing effects of betamethasone on human glioma cell line U251
}

\author{
T. Jin' , Y.X. Wang' ${ }^{2}$ K. Fan ${ }^{3}$, D.B. Tao ${ }^{2}$, X. Dong² and J.S. Shen ${ }^{2}$ \\ ${ }^{1}$ The First Affiliated Hospital of Liaoning Medical University, Jinzhou, China \\ ${ }^{2}$ Department of Neurology, \\ The First Affiliated Hospital of Dalian Medical University, Dalian, China \\ ${ }^{3}$ Department of Anatomy, Dalian Medical University, Dalian, China \\ Corresponding authors: T. Jin / Y.X. Wang \\ E-mail: netjintinglmu@yeah.net/wangyxdn@yeah.net \\ Genet. Mol. Res. 14 (3): $7841-7849$ (2015) \\ Received November 10, 2014 \\ Accepted March 12, 2015 \\ Published July 14, 2015 \\ DOI http://dx.doi.org/10.4238/2015.July.14.10
}

\begin{abstract}
We studied the differentiation-inducing effect of betamethasone on human glioma cell line U251 cultured in vitro, and the underlying mechanism. U251 cells were divided into two groups: control group cells, cultured in Dulbecco's Modified Eagle's medium containing $10 \%$ fetal bovine serum; and medication group cells, treated with $15 \mu \mathrm{M}$ betamethasone. Morphological cell changes were observed by inverted microscope, cell cycle changes were ascertained by flow cytometry, and vimentin expression was checked by immunocytochemistry. The expression levels of extracellular signal-regulated protein kinase (ERK), phosphorylated ERK (pERK), and glial fibrillary acidic protein (GFAP) were assessed by western blot. Compared with the control group, U251 cell processes increased significantly, but declined 96 $\mathrm{h}$ after betamethasone took effect. After $48 \mathrm{~h}$, the percentage of S-phase cells decreased significantly ( 28.77 to $20.42 \%$; $\mathrm{P}=0.014)$; the percentage of strongly positive vimentin cells decreased significantly (91 to $51 \% ; \mathrm{P}=0.0092)$; and the ratio of expression of GFAP protein to the internal control $\beta$-actin increased significantly $(0.24$ to $0.53 ; \mathrm{P}=0.1)$. The level of ERK protein did not change significantly 48 and $96 \mathrm{~h}$ after
\end{abstract}


the action of betamethasone, and the pERK/ERK ratios were 0.37 and 0.23 , respectively, which were significantly reduced compared with the control group $(\mathrm{P}=0.028$ and 0.006$)$. Betamethasone has a significant effect on the induction and differentiation of U251 cells, and its mechanism may be related to the inhibition of the abnormal activation of the ERK signal pathway.

Key words: Betamethasone; Glioma; Differentiation; ERK; Extracellular signal-regulated protein kinase

\section{INTRODUCTION}

Glioma is a primary tumor, the most common in the nervous system, and is difficult to treat. The current therapeutic approach is mainly surgical resection supplemented with conventional radiotherapy or chemotherapy. Patients with glioma often have a poor prognosis (Tonn et al., 2012). Induced differentiation is a new therapy, and an effective and safe drug for the treatment of glioma is eagerly sought (Rahman et al., 2012). Experiments have indicated that betamethasone, a new anticancer drug, has a differentiation-inducing effect on a variety of tumor cells. However, there are few reports on whether it can induce the differentiation in glioma cells. In this study, betamethasone was used to treat human cerebral glioma U251 cells to determine changes in the level of glioma cell differentiation and to investigate the possible mechanism by which they occur, with the aim of providing an experimental basis for clinical treatment.

\section{MATERIAL AND METHODS}

\section{Cell culture}

Human glioma U251 cells, which were purchased from the Institute of Microbiology, Chinese Academy of Sciences, were cultured in Dulbecco's Modified Eagle's Medium (DMEM) (Gibco, New York, NY, USA) containing $10 \%$ fetal bovine serum at $37^{\circ} \mathrm{C}$ in a $5 \%$ $\mathrm{CO}_{2}$ incubator. The cells were used when they reached the logarithmic growth phase.

\section{Cell morphology}

U251 cells in the logarithmic growth phase were obtained for serum starvation synchronization for $24 \mathrm{~h}$. The cells were divided into a control group and a medication group. The control group cells were routinely cultured in DMEM containing $10 \%$ fetal bovine serum (FBS), and the medication group cells were treated with $15 \mu \mathrm{M}$ betamethasone. The morphological changes in the glioma cells were observed using an inverted microscope.

\section{Cell cycle}

U251 cells were seeded into culture bottles at a density of $3 \times 10^{6}$ cells $/ \mathrm{L}, 10 \mathrm{~mL}$ to each bottle, for $24 \mathrm{~h}$ of serum starvation. The cells were divided into a control group and a medication group. The control group cells were routinely cultured in DMEM containing $10 \%$ FBS. The medication group cells were treated with $16 \mu \mathrm{M}$ betamethasone for $48 \mathrm{~h}$, digested 
with $0.125 \%$ trypsin, rinsed repeatedly to disaggregate to single cells, washed with phosphatebuffered saline, centrifuged twice to adjust the cell number of each sample to $1 \times 10^{5}$, and fixed in $75 \%$ cold ethanol at $4{ }^{\circ} \mathrm{C}$ overnight. Flow cytometry was used to detect the cells and analyze the percentage changes in the $\mathrm{G} 0 / \mathrm{G} 1, \mathrm{~S}$, and $\mathrm{G} 2 / \mathrm{M}$ phases.

\section{Vimentin expression}

U251 cells were seeded on six-well plates with $1 \times 10^{5}$ cells/well, and then starvation synchronization was conducted for $24 \mathrm{~h}$ in serum-free medium after adhesion. The cells were divided into a control group and a medication group. The control group cells were routinely cultured in DMEM containing 10\% FBS, and the medication group cells were treated with $15 \mu \mathrm{M}$ betamethasone for $48 \mathrm{~h}$. Cell culture was terminated after $48 \mathrm{~h}$ and the culture solution was discarded. The cells were fixed in $4 \%$ paraformaldehyde for $30 \mathrm{~min}$, cell membranes were ruptured using $0.15 \%$ TrintonX 100 (Jimei Bio, Shandong, China) for $30 \mathrm{~min}$, the cells were sealed with $3 \%$ hydrogen peroxide deionized water for $30 \mathrm{~min}$, rat anti-human vimentin monoclonal antibody was added, and the cells were kept at room temperature for $3 \mathrm{~h}$. The operations were performed in accordance with the immunohistochemical two-step general kit manufacturer instructions. Finally, 3,3'-diaminobenzidine staining, counterstaining, dehydration, and sealing were carried out. Five high-power fields were randomly selected to count the total number of cells and the number of positive cells, and the percentage of positive cells was calculated using the formula: percentage of positive cells $=$ number of positive cells $/$ total number of cells x 100 .

\section{Expression levels of glial fibrillary acidic protein (GFAP), extracellular signal-reg- ulated protein kinase (ERK), and phosphorylated ERK (pERK)}

U251 cells were starved of serum for $24 \mathrm{~h}$. The cells were divided into a control group and a medication group. The control group cells were culture in DMEM medium containing $10 \%$ FBS. The medication group cells were treated with $15 \mu \mathrm{M}$ betamethasone for 24,48 , and $96 \mathrm{~h}$, then collected to extract total protein and measure protein concentration. A separation gel $(4 \%)$ and a spacer gel $(10 \%)$ were prepared. Protein $(25 \mu \mathrm{g})$ was taken from each group and diluted with sample loading buffer. When the bromophenol blue had reached the junction between the separation gel and the spacer gel by electrophoresis at $60 \mathrm{~V}$ constant voltage, $100 \mathrm{~V}$ constant voltage was applied until the bromophenol blue reached the lower edge of the separation gel. The protein was transferred to a nitrocellulose membrane at $1 \mathrm{~mA} / \mathrm{cm}^{2}$ constant current, and the primary antibodies (rabbit anti-human ERK antibody, rat anti-human pERK antibody, rabbit anti-human GFAP polyclonal antibody, and rat anti-human $\beta$-actin polyclonal antibody) were added overnight. The operations were conducted in accordance with the KPL kit manufacturer instructions. Finally, exposure, development, and photographic fixing were carried out. The BandScan software was used for the analysis of protein bands.

\section{Statistical analysis}

All data were analyzed by SPSS16.0 using the $t$-test and the $\chi^{2}$ test. $\mathrm{P}<0.05$ was considered to be statistically significant. All experiments were performed in triplicate. 


\section{RESULTS}

\section{Cell morphology changes}

The inverted microscope revealed that in the control group, the U251 cells were mainly round or shaped like long spindles, had small cell bodies, had few and short processes, rarely underwent mitosis, and showed imbricated growth when growing vigorously; there was no significant contact inhibition. In the medication group, the U251 cells became polygonal or changed to resemble long spindles, the cytoplasm increased, the cell bodies enlarged, the nuclear-cytoplasm ratio decreased, processes increased, morphology became slender $96 \mathrm{~h}$ after culture with $16 \mu \mathrm{M}$ betamethasone, there was contact inhibition, and the degree of differentiation increased significantly (Figure 1).

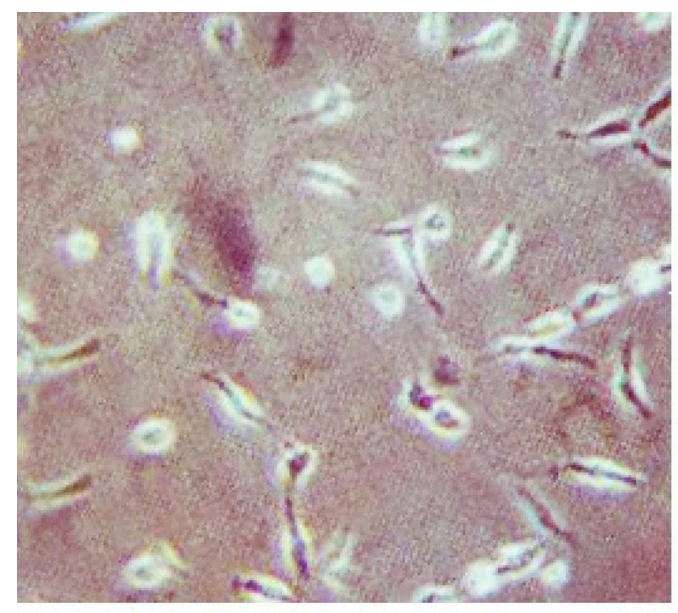

A

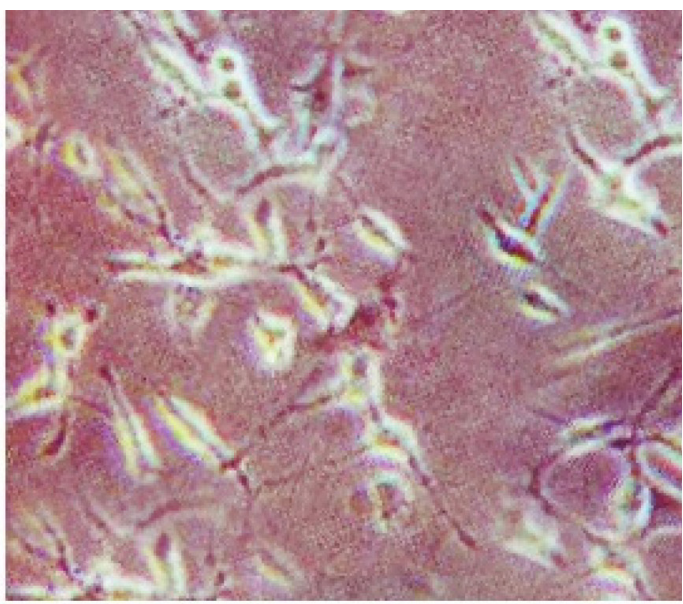

B

Figure 1. Changes in cell morphology induced by betamethasone. A. Control group; B. medication group.

\section{Cell cycle}

The results of flow cytometry showed that in the control group, $58.86 \%$ of the cells were in the $\mathrm{G} 0 / \mathrm{G} 1$ phase, $12.65 \%$ in the $\mathrm{G} 2 / \mathrm{M}$ phase, and $28.77 \%$ in the S phase. In the medication group, $68.40 \%$ of the cells were in the $\mathrm{G} 0 / \mathrm{G} 1$ phase, $11.43 \%$ in the $\mathrm{G} 2 / \mathrm{M}$ phase, and $20.42 \%$ in the S phase $48 \mathrm{~h}$ after culture with $15 \mu \mathrm{M}$ betamethasone. The difference in the percentage of cells in the $\mathrm{S}$ phase between the two groups was statistically significant $(\mathrm{P}=0.014)$.

\section{Expression levels of vimentin and GFAP}

The results of immunocytochemistry showed that vimentin protein resided in the cytoplasm, and 3,3'-diaminobenzidine-positive cells were stained yellow, clay-colored, and chocolate brown (weak positive staining was yellow and strong positive staining was chocolate brown). Cells exhibiting strong positive vimentin protein staining accounted for $91 \%$ in 
the control group and $51 \%$ in the medication group (following $48 \mathrm{~h}$ of $15 \mu \mathrm{M}$ betamethasone treatment). The difference in the percentage of cells exhibiting strong positive vimentin staining between the two groups was statistically significant $(\mathrm{P}=0.0092)$. The results of the western blot showed that the GFAP/ $\beta$-actin ratio was 0.24 in the control group, and $0.35,0.53$, and 0.68 after 24,48 , and $96 \mathrm{~h}$ of culture with $16 \mu \mathrm{M}$ betamethasone, respectively, in the medication group. The expression differences between the two groups were statistically significant ( $\mathrm{P}$ values were $0.028,0.1$, and 0.0048 , respectively) (Figures $2 \mathrm{~A}$ and B, 3, and 4).

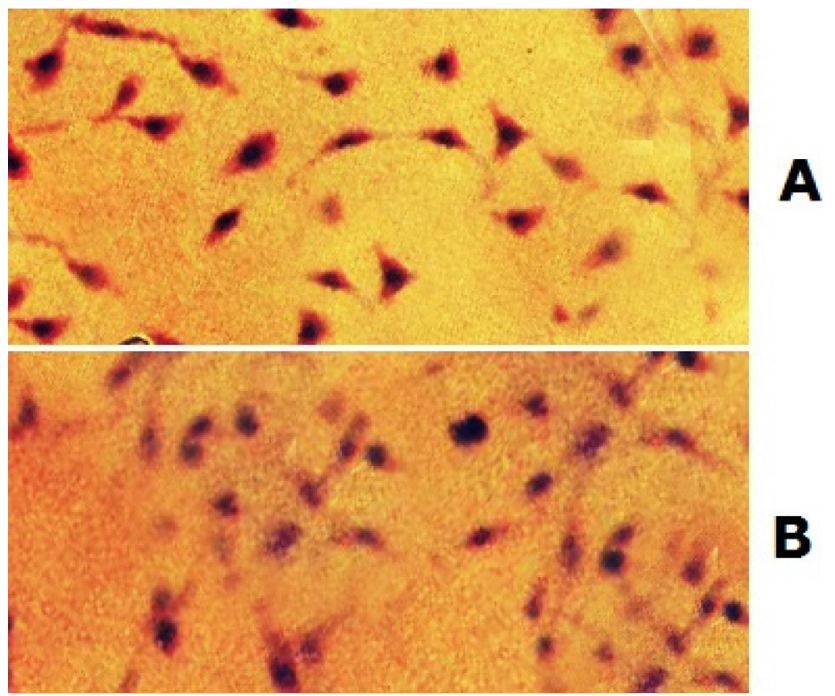

Figure 2. Immunocytochemical results of U251 cell changes induced by betamethasone. A. Control group; B. medication group.

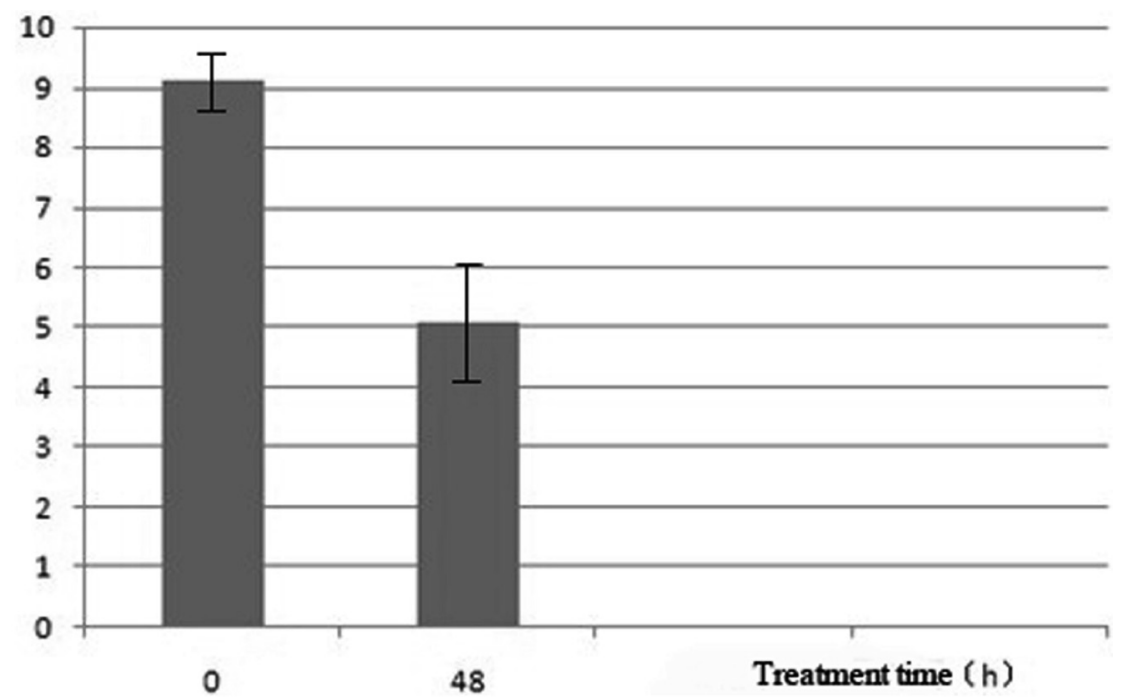

Figure 3. Positive rates of vimentin expression after treatment with betamethasone for 0 and $48 \mathrm{~h}$. 


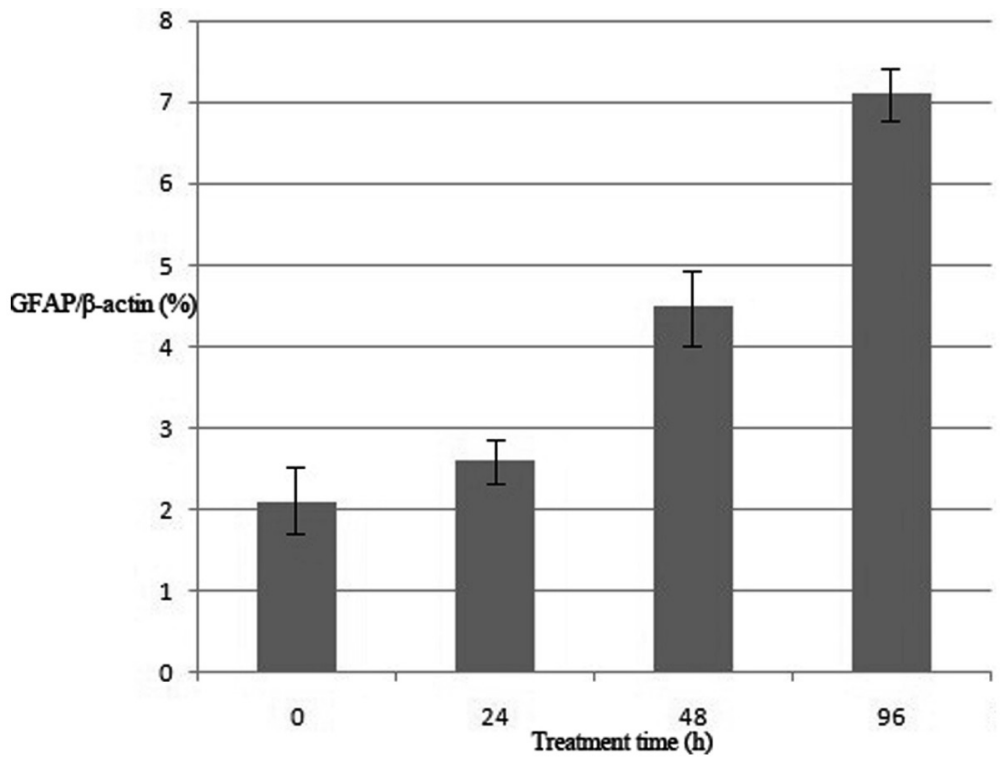

Figure 4. Effects of betamethasone on the expression of glial fibrillary acidic protein (GFAP).

\section{Expression of pERK}

The results of the western blot showed that the pERK/ERK ratio was 0.47 in the control group. The ERK protein expression was not significantly changed 24, 48, and $96 \mathrm{~h}$ after the action of $15 \mu \mathrm{M}$ betamethasone on U251 cells in the medication group. Compared with the control group, the differences were not statistically significant. pERK expression gradually decreased with time, and the pERK/ERK ratios were 0.44, 0.37, and 0.23 after 24, 48, and 96 $\mathrm{h}$ of treatment, respectively. There was no statistically significant difference between the $24 \mathrm{~h}$ group and the control group $(\mathrm{P}=0.079)$; the differences between the 48 and $96 \mathrm{~h}$ groups and the control group were statistically significant $(\mathrm{P}=0.028$ and 0.006 , respectively) (Figure 5).

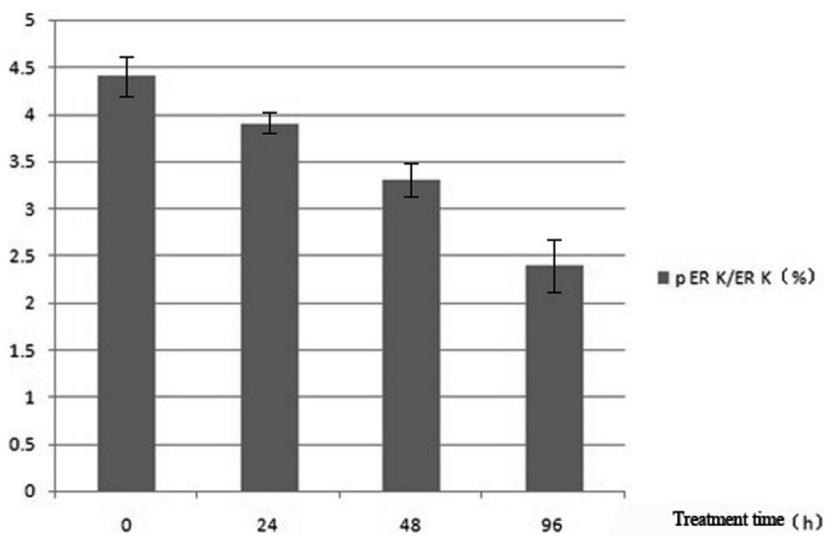

Figure 5. Effects of betamethasone on the expression of phosphorylated extracellular signal-regulated protein kinase (pERK). 


\section{DISCUSSION}

In recent years, studies on glioma have found that most of the tumor cells have limited capabilities of division and directed differentiation, but a small number of stem cells have the potential for infinite proliferation and multi-directional differentiation. Therefore, for the latter, induced differentiation therapy may help in the treatment of glioma (Filatova et al., 2013). Induced differentiation transforms tumor cells into relatively mature cells by inducing their transition from poor differentiation to high differentiation states, and restoring the functions of normal cells, thereby producing an anti-tumor effect. Recently, considerable success has been achieved in the treatment of blood diseases using malignant tumor-induced differentiation therapy. Many research institutions, both Chinese and international, have effectively screened differentiation inducers for glioma, such as retinoic acid and valproic acid (Verreault et al., 2012). However, the clinical application of most inducers is restricted owing to serious side effects. Both betamethasone and dexamethasone are long-acting hormones, but the former is cheaper because its synthesis process is easier. Betamethasone, a glucocorticoid steroid with a relatively strong anti-inflammatory effect, is effective in tumor prevention and treatment, and therefore has therapeutic potential. In this experiment, studies on various indicators confirmed that betamethasone had a differentiation-inducing effect on glioma cells.

Morphological change is the most intuitive indicator of a change in the degree of cell differentiation. The higher the degree of differentiation in tumor cells, the more closely they resemble normal cells in shape. Normal cells are characterized by adherent growth and growth termination after being merged into a single layer during in vitro culture, namely the phenomenon of contact inhibition. In contrast, tumor cells are not subject to contact inhibition and can still grow, even if they accumulate in flocks. U251 cells are human cerebral glioblastoma cells with a high degree of malignancy and poor differentiation; their morphologic characteristics are mainly manifested as different sizes and shapes, fewer and shorter processes, contact inhibition loss, infinite proliferation, layer-upon-layer accumulation, and clumped distribution in a nutritionally adequate environment. After treatment with betamethasone, the U251 cells gradually became polygonal, their volume increased, the nuclear-cytoplasm ratio decreased, processes increased in number and length, they exhibited contact inhibition, their degree of differentiation increased significantly, and they increasingly resembled normal glial cells. These were general morphologic changes observed by light microscope. The results would be more convincing using an electron microscopy to observe ultrastructural changes (Lee et al., 2008).

Cell cycle is an important manifestation of the state of cell differentiation. Most proliferating cells are in the $\mathrm{S}$ and G2 phases. With the continued growth and differentiation of cells, cell proliferation gradually decreases. This is reflected in the cell cycle; the cells mostly enter the G0/G1 phase (Malumbres, 2012). When the cell cycle is arrested in the G0/G1 phase, the cells may appear to be undergoing differentiation or apoptosis, may re-enter the cell cycle, or may be involved in many other processes (Koso et al., 2012). Therefore, cell cycle arrest is one of the important features of cell differentiation. The experimental results showed that the S-phase U251 cells decreased and the proportion of cells in the G0/G1 phase increased after betamethasone treatment. This suggests that there was synthesis disturbance of the cell DNA, the cell cycle was arrested in the G1 phase, and cells had exited the proliferation cycle and prepared for transition to the differentiation state.

Biochemical change is the most reliable indicator of cell differentiation change. Previ- 
ous studies have shown that the coexistence and expression of GFAP and vimentin in astrocyte-sourced tumors is a specific marker for astrocytes and tumors, as well as a reliable indicator to determine the degree of differentiation (Du et al., 2012). GFAP is positively correlated, and vimentin is negatively correlated, with the degree of differentiation.

In this experiment, after the treatment with betamethasone, the GFAP protein expression in U251 cell cytoplasm increased, while the expression of vimentin protein decreased, which indicates an increase in the degree of cell differentiation, consistent with the observed changes in morphology and cell cycle. It is therefore clear that betamethasone can promote the transformation of U251 cells into a benign state. The proliferation and differentiation of tumor cells are closely related. Malignant cells are characterized by high proliferation and poor differentiation. In contrast, benign cells feature lower proliferation and a higher degree of differentiation, which indicates that a dynamic equilibrium exists between the two states (Qi et al., 2012). Based on the above experimental results, betamethasone inhibited the expression of proliferation-related genes, which was manifested in morphological changes and contact inhibition, and was reflected in the cell cycle: S-phase cells decreased and G0/G1-phase cells increased. In contrast, the expression of cell differentiation-associated genes changed, manifested as the increase in GFAP protein synthesis, the decrease in vimentin protein synthesis, and an increase in cell differentiation. Betamethasone can inhibit proliferation and induce differentiation in glioma cells.

The current study showed that cell differentiation is a complex process with multiple stages, and may be related to multiple intracellular signal pathways, such as the ERK, PI3, K2A, KT, and Wnt signal pathways (Tomiyama et al., 2010). Of these pathways, the ERK signal pathway has the closest relationship with the proliferation and differentiation of tumor cells, and its activation is negatively correlated with tumor cell proliferation. Many drugs promote the differentiation of tumor cells through the inhibition of the ERK signal pathway (Gupta and Salunke, 2012). Therefore, we studied the changes of expression in the ERK signal pathway in the course of betamethasone treatment. The results showed that by acting on U251 cells, betamethasone can cause an apparent blockade of the ERK signal pathway, which was manifested as a significant decrease of expression of the active form of ERK-pERK. Therefore, the ERK signal pathway may be involved in betamethasoneinduced U251 cell differentiation (Chen et al., 2013). Whether other signal pathways are involved warrants further study.

With regards to the changes in the ERK signal pathway, many studies have found that the pathway changes induced by the action of treatment factors on cells are mainly reflected in the temporary rise or decline of the level of ERK phosphorylation within seconds to a few minutes, or in continuous changes over a few hours to several days. Different treatment factors result in different changes in the ERK pathway. Changes in the ERK pathway may even differ for a particular treatment factor, depending on the concentration used, the culture conditions, or the types of cells involved (Tsai et al., 2012). Betamethasone $(5 \mu \mathrm{M})$, inhibited the ERK signal pathway in U251 cells in a sustained way; there was no significant change in the first 24 $\mathrm{h}$ but the inhibitory effect was gradually manifested after $48 \mathrm{~h}$. Accordingly, the cell cycle and the expression of GFAP and vimentin proteins changed significantly $48 \mathrm{~h}$ after betamethasone acted on the glioma cells; the cell shape changed to a well-differentiated state after $96 \mathrm{~h}$, which suggests that betamethasone-induced differentiation is time-dependent to some extent, and could last for a relatively long time. Elucidation of the mechanisms by which betamethasone affects glioma cells warrant further study. 


\section{Conflicts of interest}

The authors declare no conflict of interest.

\section{REFERENCES}

Chen X, Zang W, Xue F, Shen Z, et al. (2013). Bioinformatics analysis reveals potential candidate drugs for different subtypes of glioma. Neurol. Sci. 34:1139-1143.

Du W, Zhou JR, Wang DL, Gong K, et al. (2012). Vitamin K1 enhances sorafenib-induced growth inhibition and apoptosis of human malignant glioma cells by blocking the Raf/MEK/ERK pathway. World J. Surg. Oncol. 10: 60.

Filatova A, Acker T and Garvalov BK (2013). The cancer stem cell niche(s): the crosstalk between glioma stem cells and their microenvironment. Biochim. Biophys. Acta. 1830: 2496-2508.

Gupta K and Salunke P (2012). Molecular markers of glioma: an update on recent progress and perspectives. J. Cancer Res. Clin. Oncol. 138: 1971-1981.

Koso H, Takeda H, Yew CC, Ward JM, et al. (2012). Transposon mutagenesis identifies genes that transform neural stem cells into glioma-initiating cells. Proc. Natl. Acad. Sci. U. S. A. 109: E2998-E3007.

Lee SA, Lee SY, Cho IH, Oh MA, et al. (2008). Tetraspanin TM4SF5 mediates loss of contact inhibition through epithelialmesenchymal transition in human hepatocarcinoma. J. Clin. Invest. 118: 1354-1366.

Malumbres M (2012). Cell cycle-based therapies move forward. Cancer Cell 22: 419-420.

Qi S, Song Y, Peng Y, Wang H, et al. (2012). ZEB2 mediates multiple pathways regulating cell proliferation, migration, invasion, and apoptosis in glioma. PLoS One 7: e38842.

Rahman M, Hoh B, Kohler N, Dunbar EM, et al. (2012). The future of glioma treatment: stem cells, nanotechnology and personalized medicine. Future Oncol. 8: 1149-1156.

Tomiyama A, Tachibana K, Suzuki K, Seino S, et al. (2010). MEK-ERK-dependent multiple caspase activation by mitochondrial proapoptotic Bcl-2 family proteins is essential for heavy ion irradiation-induced glioma cell death. Cell Death Dis. 1: e60.

Tonn JC, Thon N, Schnell O and Kreth FW (2012). Personalized surgical therapy. Ann Oncol. 23 (Suppl 10): 28-32.

Tsai CF, Yeh WL, Huang SM, Tan TW, et al. (2012). Wogonin induces reactive oxygen species production and cell apoptosis in human glioma cancer cells. Int. J. Mol. Sci. 13: 9877-9892.

Verreault M, Delattre JY and Idbaih A (2012). Adult diffuse glioma: genetic heterogeneity and therapeutic implications. Med. Sci. 28: 813-816. 